ARTICLE

Received 19 Dec 2012 | Accepted 15 May 2013 | Published 14 Jun $2013 \quad$ DOl: 10.1038/ncomms3009

\title{
Mechanical stimulation and solid seeding trigger single-crystal-to-single-crystal molecular domino transformations
}

Hajime Ito ${ }^{1}$, Mai Muromoto ${ }^{1}$, Sayaka Kurenuma ${ }^{1}$, Shoji Ishizaka², Noboru Kitamura ${ }^{3}$, Hiroyasu Sato ${ }^{4}$

$\&$ Tomohiro Seki ${ }^{1}$

Numerous studies have focused on the mechanical control of solid structures and phase changes in molecular crystals. However, the molecular-level understanding of how macroscopic forces affect the molecules in a solid remains incomplete. Here we report that a small mechanical stimulus or solid seeding can trigger a single-crystal-to-single-crystal transformation from a kinetically isolated polymorph of phenyl(phenyl isocyanide)gold(I) exhibiting blue photoluminescence to a thermodynamically stable polymorph exhibiting yellow emission without the need for heating or solvent. The phase transformation initiates at the location of the mechanical stimulation or seed crystal, extends to adjacent crystals, and can be readily monitored visually by the accompanying photoluminescent colour change from blue to yellow. The transformation was characterized using single crystal X-ray analysis. Our results suggest that the transformation proceeds through self-replication, causing the complex to behave as 'molecular dominoes'.

\footnotetext{
${ }^{1}$ Division of Chemical Process Engineering \& Frontier Chemistry Center (FCC), Graduate School of Engineering, Hokkaido University, Sapporo 060-8628, Japan. ${ }^{2}$ Department of Chemistry, Graduate School of Science, Hiroshima University, Higashi-Hiroshima 739-8526, Japan. ${ }^{3}$ Department of Chemistry, Faculty of Science, Hokkaido University, Sapporo, Hokkaido 060-0810, Japan. ${ }^{4}$ Rigaku Corporation, Akishima, Tokyo 196-8666, Japan. Correspondence and requests for materials should be addressed to H.I. (email: hajito@eng.hokudai.ac.jp).
} 
S ingle-crystal-to-single-crystal (SCSC) transformations in molecular crystals are known to be induced by various external perturbations, such as heat, light, static pressure and exposure to small molecules ${ }^{1-11}$. The crystal structures can be compared before and after the structure change using singlecrystal X-ray diffraction (XRD) analysis, providing a molecularlevel understanding of the solid-state reactions and phase transformations ${ }^{12,13}$. Anisotropic addition of a bulk force, such as shearing, ball-milling or grinding, can also alter the solid structure of the molecular crystals, and numerous studies have suggested that this transformation can include direct crystal-tocrystal conversion ${ }^{14-21}$. However, anisotropic forces also induce crystal collapse, making them unsuitable for single-crystal X-ray analysis. To date, the observation of a SCSC transformation induced by a mechanical stimulus has not yet been reported.

In this paper, we report the first observation of the SCSC transformation of phenyl(phenyl isocyanide)gold(I) (1), which was triggered by applying a small mechanical force to the crystal surface or by contact with a crystal seed of the opposite polymorph. The phase change first occurred at the initial contact area and subsequently progressed spontaneously throughout the entire crystal. Single crystal X-ray analysis was performed on the resulting crystals, providing molecular-level information on the phase transformation. Distinctive structural changes were observed, including a transition from $\mathrm{CH} / \pi$ to aurophilic intermolecular interactions ${ }^{23-25}$. The phase change was accompanied by a drastic luminescence colour change caused by the switching of the intermolecular interactions. This colour change allowed the progression of the phase change to be clearly visualized.

\section{Results}

Synthesis of 1 and its polymorph $\mathbf{I}_{\mathbf{b}}$. During the course of our studies on the luminescence mechanochromism of gold(I) complexes $^{14}$, we synthesized 1 in high yield (89\%) through the reaction of chloro(phenyl isocyanide)gold(I) with phenyllithium for $1 \mathrm{~h}$ at $-78^{\circ} \mathrm{C}$ (Fig. 1a). Careful but rapid crystallization from a hexane/dichloromethane solution produced crystals of $\mathbf{1}$ in the $\mathbf{I}_{\mathbf{b}}$ phase. Blue photoluminescence was observed from $\mathbf{I}_{\mathbf{b}}$ crystals under ultraviolet irradiation with emission maxima at 460 and $490 \mathrm{~nm}$ (quantum yield $\Phi^{\mathrm{em}}=0.15$, lifetime: $\tau_{1}=1.47 \mu \mathrm{s}$ $\left.\left(A_{1}=0.92\right), \quad \tau_{2}=72.1 \mu \mathrm{s} \quad\left(A_{1}=0.08\right), \quad \lambda_{\mathrm{ex}}=355 \mathrm{~nm}\right) \quad$ (Fig. $1 \mathrm{~b}$, blue solid line; Fig. 2a). The excitation spectrum of $\mathbf{I}_{\mathbf{b}}$ displayed a peak at $315 \mathrm{~nm}$ (Fig. 1b, blue dotted line). The crystal structure of $\mathbf{I}_{\mathbf{b}}$ was determined by the XRD of a single crystal (Fig. 2a-d; Supplementary Fig. S1; Supplementary Tables S1 and 2). $\mathbf{I}_{\mathbf{b}}$ was found to be triclinic $(P-1, a=6.0214(5) \AA, \quad b=9.0729(8) \AA$, $c=11.4498(10) \AA, \alpha=102.159(7)^{\circ}, \beta=101.468(7)^{\circ}, \gamma=102.358(7)^{\circ}$ at $123 \mathrm{~K}, Z=2, R_{1}=3.3 \%,{ }_{\mathrm{w}} R_{2}=8.2 \%$, goodness of fit $(\mathrm{GOF})=1.056$, calculated density: $\left.2.169 \mathrm{~g} \mathrm{~cm}^{-3}\right)$. X-ray analysis, elemental analysis, thermal gravimetric analysis and ${ }^{1} \mathrm{H}$ NMR measurements of $\mathbf{I}_{\mathbf{b}}$ indicated that there was no solvent inclusion in the crystals (Supplementary Figs S2 and S3; Supplementary Table S3; Supplementary Note 1). Each of the molecules in $\mathbf{I}_{\mathbf{b}}$ formed a herringbone-like structure with a headto-tail arrangement of the phenyl ligand on the gold atoms, and the isocyanide phenyl moiety twisted with a dihedral angle of $71.6^{\circ}$ (Fig. 2b) $)^{22}$. There were large distances $(>4.65 \AA$ ) between the gold atoms of adjacent molecules, indicating the absence of aurophilic interactions, which typically possess $\mathrm{Au}$...Au distances of $<3.5 \AA$ in solid-state structures ${ }^{23,24}$. Time-dependent density functional theory calculations were performed based on the crystal structure of $\mathbf{I}_{\mathbf{b}}$, affording insight into its excitation by metal-perturbed ligand-to-ligand charge transfer (Supplementary Figs S4-S8; Supplementary Tables S4-S6). a
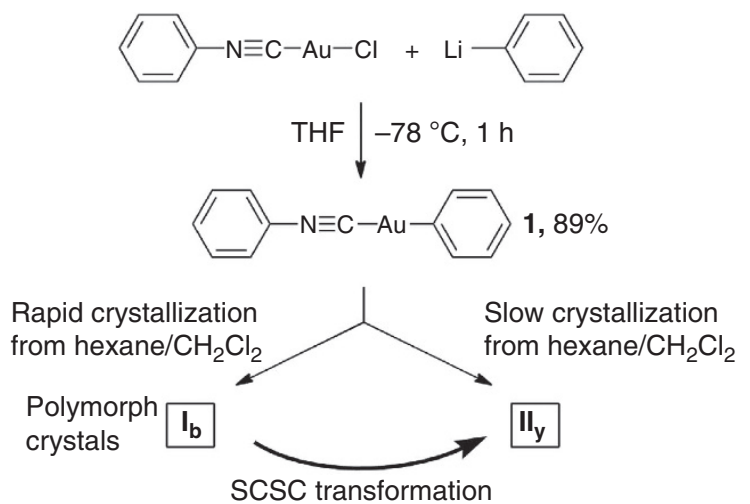

Tirggered by mechanical stimulus, or solid seeding
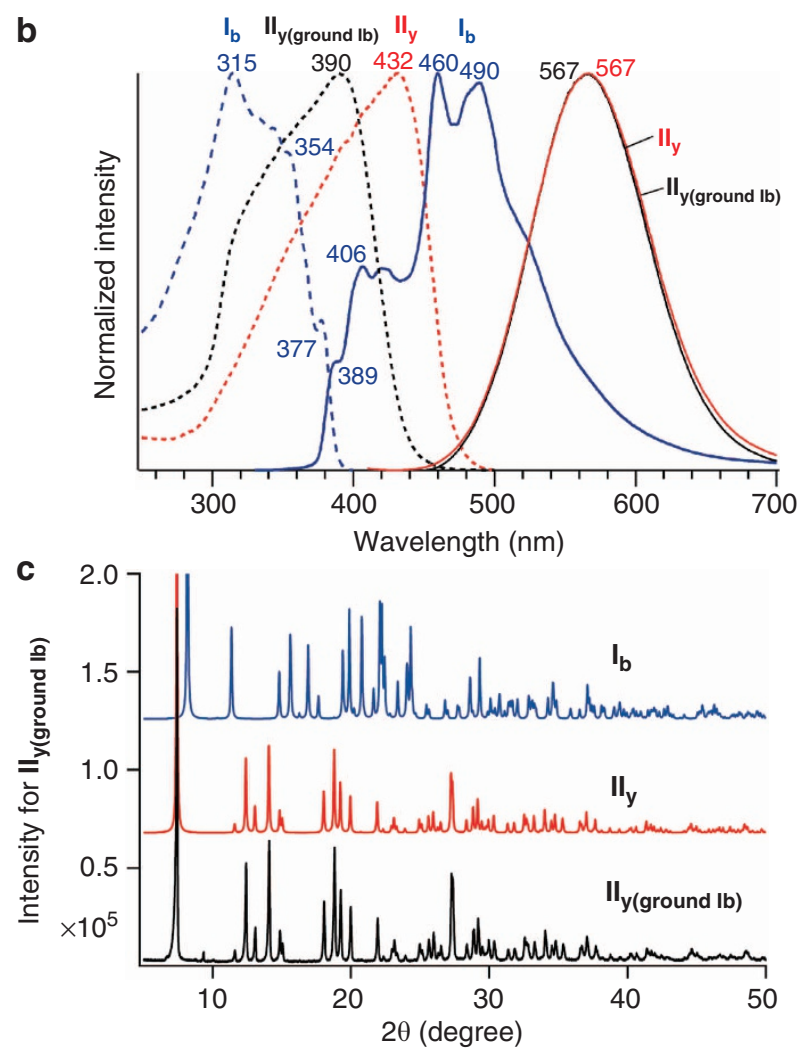

Figure 1 | Structural and optical properties of 1. (a) Synthesis, crystallization and phase conversions of 1. (b) Emission and excitation spectra, displayed as solid and dotted lines, respectively. Blue: $\mathbf{I}_{\mathbf{b}}$ (excited at $315 \mathrm{~nm}$ for the emission spectrum, observed at $460 \mathrm{~nm}$ for the excitation spectrum), red: $\mathbf{I I}_{\mathbf{y}}$ (excited at $432 \mathrm{~nm}$, observed at $565 \mathrm{~nm}$ ), black:

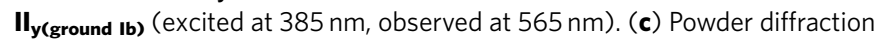
of $\mathbf{I}_{\mathbf{y}(\text { ground } \mathbf{~ b})}$ (black line), and simulated diffraction patterns for $\mathbf{I}_{\mathbf{y}}$ (red line) and $\mathbf{I}_{\mathbf{b}}$ (blue line).

Solution phase preparation of polymorph $\mathrm{II}_{\mathrm{y}}$. The slow crystallization of $\mathbf{1}$ from a hexane/dichloromethane solution produced the polymorphic crystals $\mathbf{I I}_{\mathbf{y}}$, which exhibited a significantly different crystal structure compared with that of $\mathbf{I}_{\mathbf{b}}$. The $\mathbf{I I}_{\mathbf{y}}$ crystals exhibited a strong yellow emission $\left(\lambda_{\max }=571 \mathrm{~nm}\right)$ under ultraviolet irradiation $(365 \mathrm{~nm}$ ) (Fig. 1b, red solid line and Fig. 2e). A higher emission quantum yield $\left(\Phi^{\mathrm{em}}=0.43\right)$ and shorter emission lifetime $\left(\tau_{1}=0.17 \mu \mathrm{s}\left(A_{1}=0.20\right), \tau_{2}=0.65 \mu \mathrm{s}\right.$ $\left.\left(A_{2}=0.80\right), \lambda_{\mathrm{ex}}=355 \mathrm{~nm}\right)$ were observed compared with those for $\mathbf{I}_{\mathbf{b}}$. The excitation spectrum of $\mathbf{I I}_{\mathbf{y}}$ displayed a peak at a longer wavelength $(432 \mathrm{~nm})$ than that of $\mathbf{I}_{\mathbf{b}}$ (Fig. $1 \mathbf{b}$, red dotted line). The 
a

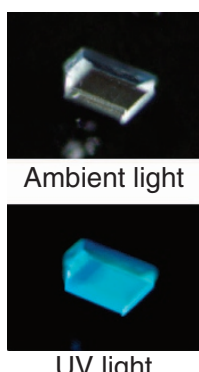

UV light

e

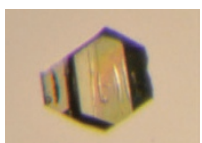

Ambient light

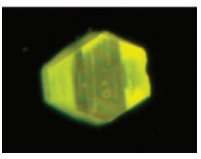

UV light b

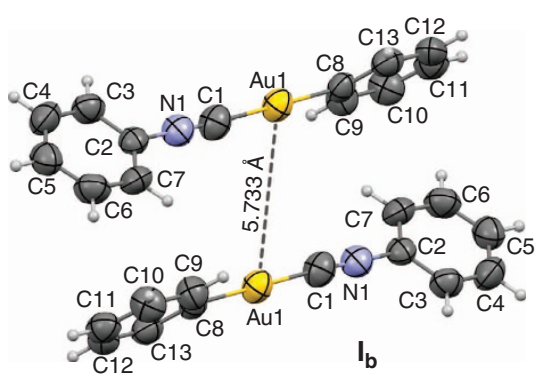

f

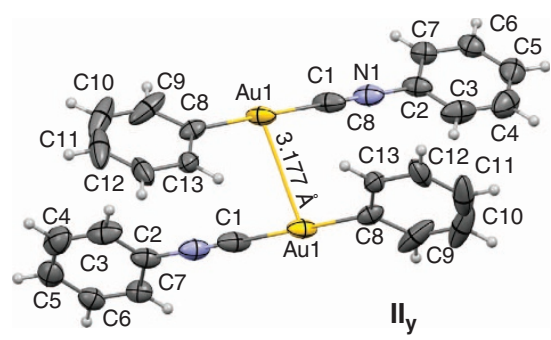

C

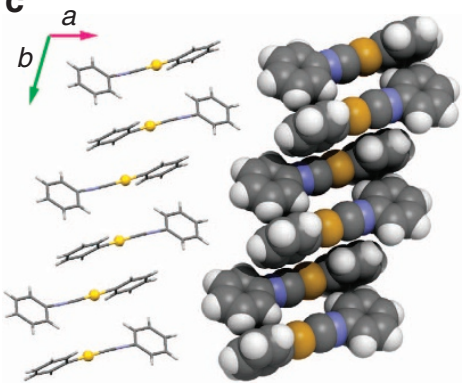

g

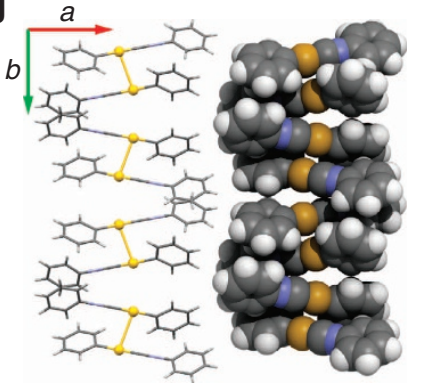

d

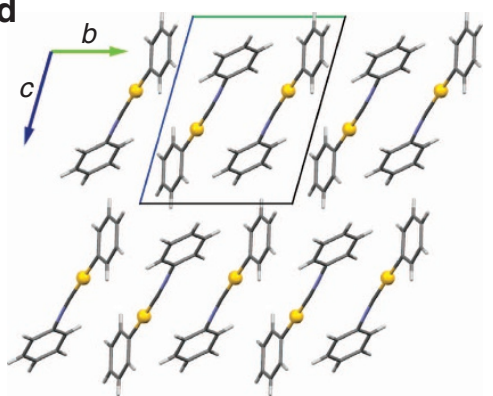

h

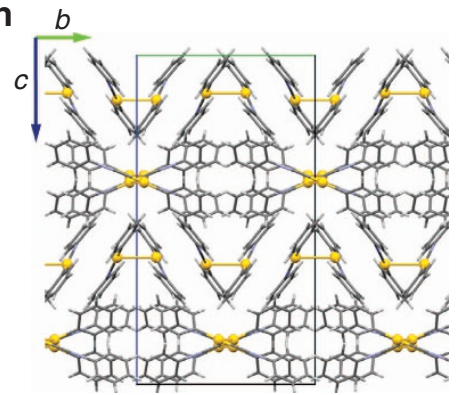

Figure 2 | Photographs and crystal structures of polymorphs $\mathbf{I}_{\mathbf{b}}$ and $\mathbf{I}_{\mathbf{y}}$. (a) Photographs of polymorph $\mathbf{I}_{\mathbf{b}}$ under ambient and ultraviolet (UV, $365 \mathrm{~nm}$ ) light. (b) Oak Ridge Thermal Ellipsoid Program (ORTEP) drawing (50\% probability level) of the crystal structure of $\mathbf{I}_{\mathbf{b}}$. (c) Crystal structure of $\mathbf{I}_{\mathbf{b}}$ depicted as stick and space filling models viewed along the $c$ axis. (d) Crystal structure of $\mathbf{I}_{\mathbf{b}}$ viewed along the $a$ axis. (e) Photographs of the polymorph $\mathbf{I I}_{\mathbf{y}}$ under ambient and UV (365 nm) light. (f) ORTEP drawing (50\% probability level) of the crystal structure of $\mathbf{I I}_{\mathbf{y}}$. (g,h) Crystal structures of $\mathbf{I}_{\mathbf{y}}$.

crystal structure of $\mathbf{I I}_{\mathbf{y}}$ was in the tetragonal system with a space group of $I-42 d$ (Fig. 2 f-h, $a=13.4781$ (4) $\AA, c=24.8079(8) \AA$ at $123 \mathrm{~K}, Z=16, R_{1}=2.5 \%,{ }_{\mathrm{w}} R_{2}=5.4 \%, \mathrm{GOF}=1.093$, calculated density: $2.224 \mathrm{~g} \mathrm{~cm}^{-3}$, Supplementary Fig. S9; Supplementary Tables S1 and S2). X-ray analysis, elemental analysis and ${ }^{1} \mathrm{H}$ NMR measurements of $\mathbf{I I}_{\mathbf{y}}$ indicated that there was no solvent inclusion in the crystals (Supplementary Fig. S2; Supplementary Table S3; Supplementary Note 1). The molecules of $\mathbf{I I}_{\mathbf{y}}$ adopted a nearly flat conformation with a dihedral angle between the isocyanide phenyl group and the gold-coordinated phenyl group of $3.1^{\circ}$. Oblique head-to-tail dimers contained a short $\mathrm{Au} \cdot \cdots \mathrm{Au}$ distance of $3.177 \AA$, suggesting the presence of significant aurophilic interactions ${ }^{23,24}$. Each dimer interacted with adjacent dimers through isocyanide carbons separated by just $3.186 \AA$ to form 1-D chain-like structures comprising dimer units (Supplementary Fig. S9). Time-dependent density functional theory calculations of a model structure based on the $\mathbf{I I}_{\mathbf{y}}$ crystals indicated a metal-metal to ligand charge transfer characteristic, which was facilitated by the short $\mathrm{Au} \cdot$. Au distance (Supplementary Figs S4, S10 and S11; Supplementary Tables S4 and S7) 23,24 .

Luminescent mechanochromism of $\mathbf{I}_{\mathbf{b}}$. The blue luminescent $\mathbf{I}_{\mathbf{b}}$ displayed a significant luminescence mechanochromism (Fig. 3a) ${ }^{14-17}$. The ball-milling of $\mathbf{I}_{\mathbf{b}}$ for $3 \mathrm{~min}$ afforded the

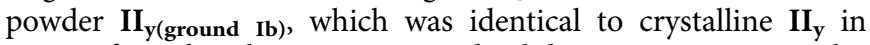
terms of its photoluminescence and solid-state structure. Similar

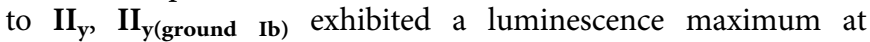
$567 \mathrm{~nm}$, an emission quantum yield of 0.38 , and emission lifetimes of $\tau_{1}=0.27 \mu \mathrm{s}\left(A_{1}=0.34\right)$ and $\tau_{2}=0.68 \mu \mathrm{s}\left(A_{1}=0.66\right)$ at an excitation wavelength of $355 \mathrm{~nm}$ (Fig. 2b, black solid line) with an excitation peak at 400nm (Fig. 2b, black dotted line). The powder XRD pattern of the ball-milled sample ( $\mathbf{I I}_{\mathbf{y}(\text { ground }} \mathbf{I b}$ ), Fig. 1c, black solid line) matched the simulated diffraction pattern based on the single crystal X-ray analysis of $\mathbf{I I}_{\mathbf{y}}$ (Fig. 1c, red solid line), and no trace of $\mathbf{I}_{\mathbf{b}}$ was found in the ball-milled sample (Fig. 1c, blue solid line). These results indicate that the ballmilling process induced direct crystal-to-crystal transformation of $\mathbf{I}_{\mathbf{b}}$ to $\mathrm{II}_{\mathbf{y}}$.

SCSC transformation. The ball-milling described above involves a considerable mechanical stimulus; we next conducted the mechanical triggering experiment with a much smaller stimulus. A small pit was formed on the surface of an $\mathbf{I}_{\mathbf{b}}$ crystal using a needle under atmospheric conditions (Fig. 3b). A yellow luminescent spot was initially observed at the location of the small pit. Subsequently, the domain exhibiting yellow emission gradually increased to nearly the entire crystal after $9 \mathrm{~h}$ at room temperature. The transformation could also be triggered by contacting a seed crystal of $\mathbf{I I}_{\mathbf{y}}$ with a crystal of $\mathbf{I}_{\mathbf{b}}$ (Fig. 3c). The luminescent colour change initiated at the point of contact and extended to the entire crystal after $15 \mathrm{~h}$. This colour change propagated through the contacting surfaces of adjacent crystals; eventually, the blue luminescent crystals that were in contact with neighbouring yellow luminescent crystals became yellow, maintaining their transparency (Fig. 3d,e, Supplementary Fig. 12). This solidseeding phase change represents a self-replicating progression of phase transformation. It should be noted that the rate of conversion was highly variable: some crystals changed rapidly, whereas others were slower.

Single crystal X-ray analysis was performed after the mechanical stimulus-triggered SCSC transformation of $\mathbf{I}_{\mathbf{b}}$. A yellow luminescent crystal was prepared by the mechanical pricking of a small region of one $\mathbf{I}_{\mathbf{b}}$ crystal. After the initial contact area was removed to avoid affecting the quality of the XRD data, the yellow emissive crystal (denoted $\mathbf{I I}_{\mathbf{y s c s c}}$ ) was subjected to single crystal $\mathrm{X}$-ray analysis. The crystal structure of $\mathbf{I I}_{\mathbf{y s c s c}}$ obtained via SCSC was identical to that of $\mathbf{I I}_{\mathbf{y}}$ crystallized from the solution phase 


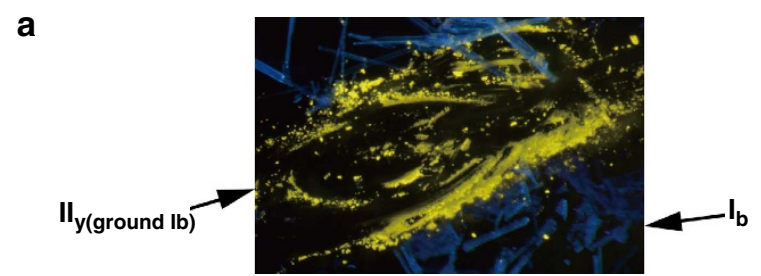

b

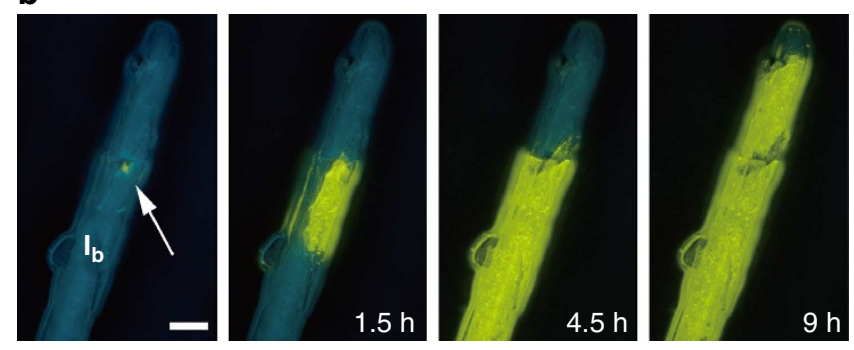

c
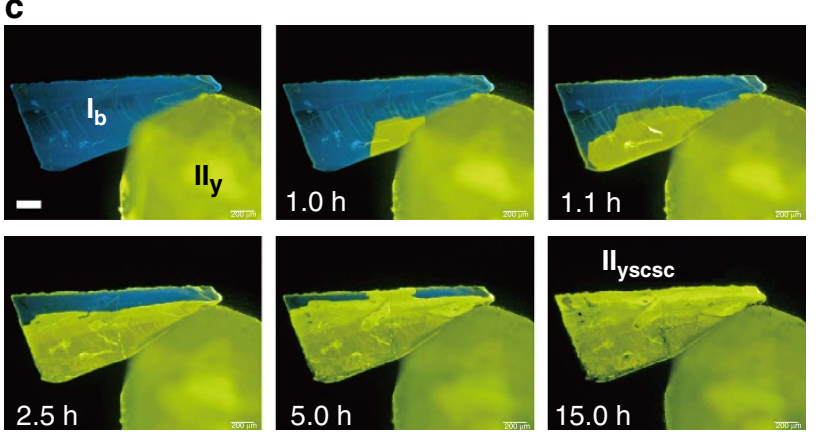

d
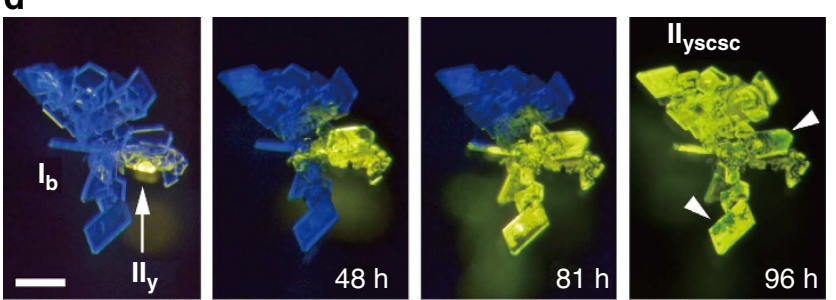

e

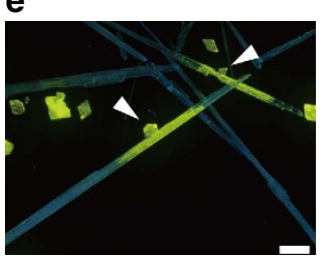

f

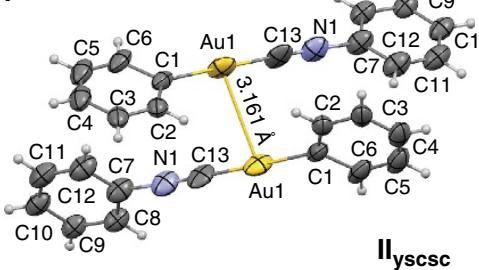

II

Figure 3 | Photographs of the phase transformation of $\mathbf{I}_{\mathbf{b}}$ to $\mathbf{I I}_{\mathbf{y}}$. (a) Luminescence mechanochromism of $\mathbf{I}_{\mathbf{b}}$. Yellow luminescent $\mathbf{I I}_{\mathbf{y}(\text { ground } \mathbf{I b})}$ was generated by grinding the blue luminescent $\mathbf{I}_{\mathbf{b}}$ powder with a spatula. (b) Mechanical stimulus-triggered phase transformation (scale bar, $200 \mu \mathrm{m}$ ). A small pit (left, white arrow) was first produced by pricking the fixed sample with a needle. The phase transformation gradually spread over the entire crystal after $9 \mathrm{~h}$. (c) Solid seeding-triggered phase transformation (scale bar, $200 \mu \mathrm{m}$ ). The phase transformation of $\mathbf{I}_{\mathbf{b}}$ initiated from the area in contact with the seed crystal of $\mathbf{I}_{\mathbf{y}}$ (upper left photograph) and spread over the entire crystal after $15 \mathrm{~h}$ (lower right photograph). (d) Progression of the phase transformation over the crystals (scale bar, $200 \mu \mathrm{m}$ ). Partial decomposition of the $\mathbf{I}_{\mathbf{y}}$ phase was observed (white triangles).

(e) Progression of phase transformation from the seed crystals (white triangles; scale bar, $200 \mu \mathrm{m}$ ). (f) Crystal structure of $\mathbf{I I}_{\mathbf{y s c s c}}$. These mechanical- and seeding-triggered phase transformation experiments could be replicated many times; however, the conversion rate varied considerably from crystal to crystal.

(Fig. 3f; Supplementary Fig. S13; Supplementary Tables S1 and

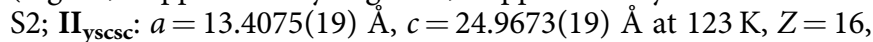
$R_{1}=8.8 \%, \quad{ }_{\mathrm{w}} R_{2}=21.7 \%, \quad \mathrm{GOF}=1.316$, calculated density: $2.233 \mathrm{~g} \mathrm{~cm}^{-3}$ ).

\section{Discussion}

The mechanical- or solid seeding-triggered phase change described in this study occurred at room temperature, whereas a thermal phase transformation of $\mathbf{I}_{\mathbf{b}}$ to $\mathbf{I I}_{\mathbf{y}}$ required elevated temperatures of $64.5-74.9^{\circ} \mathrm{C}$ (Supplementary Fig. S3). No reverse phase change was observed when the sample was cooled. The $\mathbf{I}_{\mathbf{b}}$ crystal could only be regenerated by the dissolution and recrystallization of $\mathbf{I I}_{\mathbf{y}}$. The thermal analysis suggested that $\mathbf{I}_{\mathbf{b}}$ was a metastable, kinetically isolated form of $\mathbf{1}$, and $\mathbf{I I}_{\mathbf{y}}$ was the thermodynamically favoured form (Supplementary Fig. S14). The thermodynamic analysis and the sample crystallization process revealed that $\mathbf{1}$ is a good example of the Ostwald rule: the less stable polymorphs $\left(\mathbf{I}_{\mathbf{b}}\right)$ crystallize first, and the more stable polymorphs $\left(\mathbf{I I}_{\mathbf{y}}\right)$ form later ${ }^{1-3}$. In some reports, mechanical pricking or the presence of defects can accelerate the phase change near the thermal phase change temperature, resulting in polycrystalline formation ${ }^{1,3,13,25}$. However, the phase transformation of $\mathbf{I}_{\mathbf{b}}$ occurred at much lower temperatures, suggesting that the transformation observed in our study was triggered purely by mechanical stimulation. To our knowledge, this is the first clear example of the mechanical- or solid seedingtrigged SCSC transformation of molecular crystals. In addition, the emergence of metallophilic interactions after the SCSC transformation of mother crystals lacking metallophilic interactions has not been reported previously.

The solid-state structures of $\mathbf{I}_{\mathbf{b}}$ and $\mathbf{I I}_{\mathbf{y}}$ varied greatly (triclinic $P-1$ and tetragonal $I-42 d$ ) (see Supplementary Fig. S15). Thus, the significant structural transformation cannot be explained by a simple conformational change or molecular reorientation. The phase change may proceed by a similar mechanism to the epitaxial mechanism proposed for the thermal phase transformation $^{3,25}$. A small portion of the $\mathbf{I I}_{\mathbf{y}}$ daughter phase forms initially in the $\mathbf{I}_{\mathbf{b}}$ mother phase through mechanical stress or contact with a seed crystal of $\mathbf{I I}_{\mathbf{y}}$ (Fig. 4a,b). The molecules in the thermodynamically unstable $\mathbf{I}_{\mathbf{b}}$ phase diffuse across the narrow gap between phases $\mathbf{I}_{\mathbf{b}}$ and $\mathbf{I I}_{\mathbf{y}}$, and rearrange to the $\mathbf{I I}_{\mathbf{y}}$ phase through the formation of intermolecular aurophilic interactions (Fig. 4c,d). a

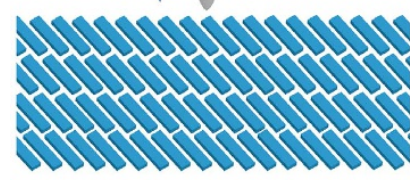

C

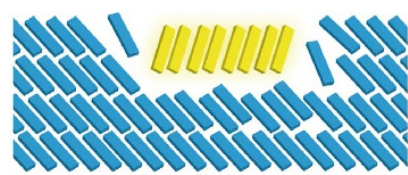

b

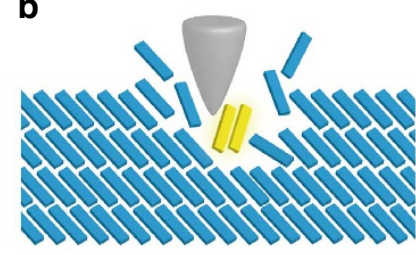

d

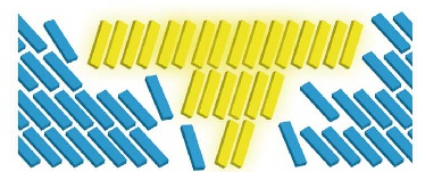

Figure 4 | Proposed mechanism for the mechanical stimulus-triggered phase transformation. (a) Mechanical stimulation of the metastable $\mathbf{I}_{\mathbf{b}}$ phase (blue rectangles). (b) Generation of the $\mathbf{I I}_{\mathbf{y}}$ phase. (c, d) The thermodynamically stable $\mathbf{I I}_{\mathbf{y}}$ phase extends by absorbing molecules from the metastable $\mathbf{I}_{\mathbf{b}}$ phase. 
This phase transformation resembles dominoes, in that a mechanical stimulus (pushing) triggers a state change in the entire assembly. The self-replicative nature of this molecular domino may provide a new way to control nano or molecular structures through macroscopic mechanical stimulation. The domino-like amplification of the optical response to the small mechanical stimulus (from the small pit to the entire crystal) can be applied to the highly sensitive detection of mechanical stimulation.

\section{Methods}

Synthesis of 1. Chloro(phenyl isocyanide)gold(I) was prepared from chloro (tetrahydrothiophene)gold(I) and phenyl isocyanide using a method similar to that reported previously ${ }^{26}$. Chloro(phenyl isocyanide)gold(I) $(100.7 \mathrm{mg}, 0.30 \mathrm{mmol})$ and a magnetic stir bar were placed in a vial and sealed with a rubber septum. After the reaction vial was connected to a vacuum manifold through a needle, it was evacuated and then back filled with nitrogen. Dry THF $(0.44 \mathrm{ml})$ was added via a syringe to dissolve the chloro(phenyl isocyanide)gold(I). A butyl ether solution of phenyllithium $(0.164 \mathrm{ml}, 1.83 \mathrm{M}, 0.30 \mathrm{mmol})$ was added at $-78^{\circ} \mathrm{C}$ under stirring. After $30 \mathrm{~min}$, the reaction was quenched by the addition of a saturated aqueous solution of $\mathrm{NH}_{4} \mathrm{Cl}(2 \mathrm{ml})$, extracted three times with $\mathrm{CH}_{2} \mathrm{Cl}_{2}$, washed with brine and then dried over $\mathrm{MgSO}_{4}$. After filtration, the volatiles were removed under reduced pressure. Crude phenyl(phenyl isocyanide)gold(I) (1) was obtained as a pale purple solid $(106.7 \mathrm{mg}, 89 \%)$. The yield was determined by ${ }^{1} \mathrm{H}$ NMR spectroscopy using $\mathrm{Cl}_{2} \mathrm{CHCHCl}_{2}$ as an internal standard. 1 was recrystallized from $\mathrm{CH}_{2} \mathrm{Cl}_{2}$ /hexane after filtration to remove an insoluble purple solid. 1 was fairly stable in air at room temperature, but readily decomposed at elevated temperatures $\left(>80^{\circ} \mathrm{C}\right)$, with metal deposition occurring on its surface. In solution, $\mathbf{1}$ remained intact in the dark for a short period $(<1 \mathrm{~h})$ but readily decomposed under light. The data for ${ }^{1} \mathrm{H}$ and ${ }^{13} \mathrm{C}$ NMR and HRMS were included in the Supplementary Methods.

Crystal and powder sample preparation $\left(\mathbf{I}_{\mathbf{b}}, \mathbf{I}_{\mathbf{y}}\right)$. Typical preparation procedures are as follows. On the surface of a solution of $\mathbf{l}$ (prepared from $\mathbf{I}_{\mathbf{b}}$ or $\mathbf{I I}_{\mathbf{y}}, 20 \mathrm{mg}$ ) in $\mathrm{CH}_{2} \mathrm{Cl}_{2}(1.0 \mathrm{ml}) /$ hexane $(2.0 \mathrm{ml})$ in a small glass vial, a stream of nitrogen gas was flowed through a needle connected to a nitrogen line for a few minutes to produce crystals of $\mathbf{I}_{\mathbf{b}}$. $\mathbf{I I}_{\mathbf{y}}$ crystals were obtained by the slow crystallization (typically $10 \mathrm{~h}$ ) of a solution of $\mathbf{1}$ in $\mathrm{CH}_{2} \mathrm{Cl}_{2}$ /hexane placed in a refrigerator. The ground sample of $\mathbf{I I}_{\mathbf{y}(\text { ground } \mathbf{~ I b})}$ was prepared with a ball mill (Taitec Bead Crusher $\mu \mathrm{T}-01$, Koshigaya, Saitama, Japan). Typically, $\mathbf{I}_{\mathbf{b}}(50 \mathrm{mg})$ was treated in a micro tube $(\phi 13 \times 49 \mathrm{~mm})$ with a screw cap at a shaking speed of $4,600 \mathrm{r} \mathrm{min}^{-1}$ for $3 \mathrm{~min}$ ( $60 \mathrm{~s}$ three times) using a stainless bead (1/8 inch).

Single crystal and powder $\mathbf{X}$-ray analysis. Measurements for $\mathbf{I}_{\mathrm{b}}, \mathrm{II}_{\mathbf{y}}$ and $\mathrm{II}_{\mathrm{yscs}}$ were made on a Rigaku R-AXIS RAPID diffractometer using graphite monochromatic Mo- $\mathrm{K}_{\alpha}$ radiation. The diffraction data were collected at $-150^{\circ} \mathrm{C}$. The structure was solved by direct methods and expanded using Fourier techniques. Non-hydrogen atoms were refined anisotropically. Hydrogen atoms were refined using the riding model. All calculations were performed using the CrystalStructure crystallographic software package, except for refinement, which was performed using SHELXL-97. The powder diffraction data for $\mathbf{I I}_{\mathbf{y}(\text { ground } \mathbf{~ I b )} \text { were recorded at }}$ room temperature on a Rigaku SmartLab diffractometer with $\mathrm{Cu}-\mathrm{K}_{\alpha}$ radiation and a D/teX Ultra detector covering $5-50^{\circ}(2 \theta)$. The diffraction data were collected at $20^{\circ} \mathrm{C}$. The powder simulation patterns for $\mathbf{I}_{\mathbf{b}}$ and $\mathbf{I I}_{\mathbf{y}}$ were generated from the single crystal X-ray structures collected at $20^{\circ} \mathrm{C}$. The powder patterns for $\mathbf{I}_{\mathbf{b}}$ and $\mathbf{I I}_{\mathbf{y}}$ displayed in Fig. 1b were generated using Mercury 3.0.

\section{References}

1. Bernstein, J. Polymorphism in Molecular Crystals (Oxford Univ. Press, 2002).

2. Wildfong, P. L. D. Polymorphism in Pharmaceutical Solids 2nd edn (Informa Healthcare, 2009).

3. Mnyukh, Y. Fundamentals of Solid-State Phase Transitions, Ferromagnetism and Ferroelectricity 2nd edn (Direct Scientific Press, 2010).

4. Kobatake, S., Takami, S., Muto, H., Ishikawa, T. \& Irie, M. Rapid and reversible shape changes of molecular crystals on photoirradiation. Nature 446, 778-781 (2007)

5. Mutai, T., Satou, H. \& Araki, K. Reproducible on-off switching of solid-state luminescence by controlling molecular packing through heat-mode interconversion. Nat. Mater. 4, 685-687 (2005).

6. Huang, Z., White, P. S. \& Brookhart, M. Ligand exchanges and selective catalytic hydrogenation in molecular single crystals. Nature 465, 598-601 (2010).

7. Malwitz, M. A. et al. Crystallization and interconversions of vapor-sensitive, luminescent polymorphs of $\left[\left(\mathrm{C}_{6} \mathrm{H}_{11} \mathrm{NC}\right)_{2} \mathrm{AuI}\right]\left(\mathrm{AsF}_{6}\right)$ and $\left[\left(\mathrm{C}_{6} \mathrm{H}_{11} \mathrm{NC}\right)_{2} \mathrm{AuI}\right]\left(\mathrm{PF}_{6}\right)$. J. Am. Chem. Soc. 134, 10885-10893 (2012).
8. Zenkina, O. V., Keske, E. C., Wang, R. \& Crudden, C. M. Double single-crystalto-single-crystal transformation and small-molecule activation in rhodium nhe complexes. Angew. Chem., Int. Ed. 50, 8100-8104 (2011)

9. Biradha, K. \& Fujita, M. A springlike 3D-coordination network that shrinks or swells in a crystal-to-crystal manner upon guest removal or readsorption. Angew. Chem. Int. Ed. 41, 3392-3395 (2002).

10. Wu, C.-D. \& Lin, W. Highly porous, homochiral metal-organic frameworks: solvent-exchange-induced single-crystal to single-crystal transformations. Angew. Chem. Int. Ed. 44, 1958-1961 (2005).

11. Albrecht, M., Lutz, M., Spek, A. \& van Koten, G. Organoplatinum crystals for gas-triggered switches. Nature 406, 970-974 (2000).

12. Halasz, I. Single-crystal-to-single-crystal reactivity: gray, rather than black or white. Cryst. Growth Des. 10, 2817-2823 (2010).

13. Desiraju, G. R., Vittal, J. J. \& Ramanan, A. Crystal Engineering: a Textbook (World Scientific; IISc Press, 2011).

14. Ito, $\mathrm{H}$. et al. Reversible mechanochromic luminescence of $\left[\left(\mathrm{C}_{6} \mathrm{~F}_{5} \mathrm{Au}\right)_{2}\right.$ ( $\mu$-1,4-diisocyanobenzene)]. J. Am. Chem. Soc. 130, 10044-10045 (2008).

15. Sagara, Y. \& Kato, T. Mechanically induced luminescence changes in molecular assemblies. Nat. Chem. 1, 605-610 (2009).

16. Ariga, K., Mori, T. \& Hill, J. P. Mechanical control of nanomaterials and nanosystems. Adv. Mater. 24, 158-176 (2012).

17. Balch, A. Dynamic crystals: visually detected mechanochemical changes in the luminescence of gold and other transition-metal complexes. Angew. Chem. Int. Edit. 48, 2641-2644 (2009).

18. Descamps, M., Willart, J. F., Dudognon, E. \& Caron, V. Transformation of pharmaceutical compounds upon milling and comilling: the role of $\mathrm{Tg}$. J. Pharm. Sci. 96, 1398-1407 (2007).

19. Brittain, H. G. Effects of mechanical processing on phase composition. J. Pharm. Sci. 91, 1573-1580 (2002).

20. Wildfong, P. L. D., Morris, K. R., Anderson, C. A. \& Short, S. M. Demonstration of a shear-based solid-state phase transformation in a small molecular organic system: chlorpropamide. J. Pharm. Sci. 96, 1100-1113 (2007).

21. Braga, D., Grepioni, F. \& Maini, L. The growing world of crystal forms. Chem. Commun. 46, 6232-6242 (2010).

22. Tiekink, E. R. T. \& Zukerman-Schpector, J. The Importance of Pi-Interactions in Crystal Engineering: Frontiers in Crystal Engineering (Wiley, 2012).

23. Schmidbaur, H. \& Schier, A. A briefing on aurophilicity. Chem. Soc. Rev. 37, 1931-1951 (2008).

24. Yam, V. W.-W. \& Cheng, E. C.-C. Highlights on the recent advances in gold chemistry - a photophysical perspective. Chem. Soc. Rev. 37, 1806-1813 (2008)

25. Herbstein, F. H. On the mechanism of some first-order enantiotropic solidstate phase transitions: from Simon through Ubbelohde to Mnyukh. Acta. Crystallogr. Sect. B. Struct. Sci. 62, 341-383 (2006).

26. Hashmi, A. S. K., Yu, Y. \& Rominger, F. Efficient one-pot synthesis of unsymmetrical gold(I) N-heterocyclic carbene complexes and their use as catalysts. Organometallics 31, 895-904 (2012).

\section{Acknowledgements}

The Funding Program for Next Generation World-Leading Researchers (NEXT Program, No. GR002) is gratefully acknowledged. We thank Professor Masaya Sawamura and Professor Yoshihide Nakao for their suggestions. We appreciate Professor Toyoji Kakuchi, Professor Toshihumi Sato and Kenta Sakurada for thier help with thermal gravimetric analysis analysis.

\section{Author contributions}

H.I. wrote this paper. S.I., N.K. and T.S. helped with the spectroscopic analysis. S.H. did the XRD measurements and analyses. M.M. and S.K. synthesized $\mathbf{1}$ and performed all the other experiments.

\section{Additional information}

Accession codes: The X-ray crystallographic coordinates for the structures reported in this article have been deposited at the Cambridge Crystallographic Data Centre (CCDC) under deposition numbers CCDC 897541, 897542 and 909344. These data can be obtained free of charge from The Cambridge Crystallographic Data Centre via www.ccdc.cam.ac.uk/data_request/cif.

Supplementary Information accompanies this paper at http://www.nature.com/ naturecommunications

Competing financial interests: The authors declare no competing financial interests.

Reprints and permission information is available online at http://npg.nature.com/ reprintsandpermissions/

How to cite this article: Ito, H. et al. Mechanical stimulation and solid seeding trigger single-crystal-to-single-crystal molecular domino transformations. Nat. Commun. 4:2009 doi: $10.1038 /$ ncomms3009 (2013) 